\title{
A note on Grayson's theorem
}

\author{
Annibale Magni(*) - Carlo Mantegazza(**)
}

ABstract - In this note we show a variational proof of Matthew Grayson's convexification theorem for simple closed curves moving by curvature in the plane.

Mathematics SubJect Classification (2010). 53C44, 35K93.

KEYWoRDS. Curve shortening flow, geometric measure theory.

\section{Contents}

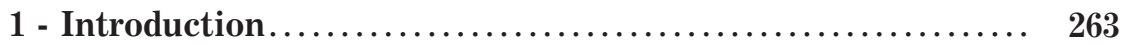

2 - Analytical and Geometrical Facts .................... 265

3 - The Density Function........................... 267

4 - Blowing-Up at a Singularity - The Proof of Grayson's Theorem 269

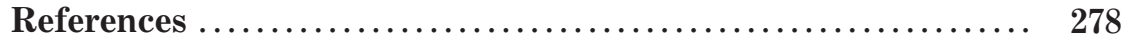

\section{Introduction}

Let $\gamma: \mathbb{S}^{1} \times[0, T) \rightarrow \mathbb{R}^{2}$ be the curvature flow of a simple smooth closed curve in the Euclidean plane, that is, satisfying $\partial_{t} \gamma=k v$, such that $T$ is the maximal time of smooth existence. In this paper we give a new proof of the following result.

(*) Indirizzo dell'A.: Albert-Ludwigs Universität Freiburg, Eckerstr. 1, Freiburg im Breisgau, Germany, 79104.

E-mail: annibale.magni@math.uni-freiburg.de

(**) Indirizzo dell'A.: Scuola Normale Superiore, Piazza dei Cavalieri 7, 56126, Pisa, Italy.

E-mail: c.mantegazza@sns.it 
Theorem 1.1 (Grayson's Theorem [11]). At some time the evolving curve becomes convex.

REMARK 1.2. After the curve has become convex, the work of Gage and Hamilton $[8,9,10]$ shows that it remains convex and it shrinks to a point of $\mathbb{R}^{2}$ in finite time, becoming asymptotically circular.

We borrow some ideas and techniques from the work of Ilmanen [16], Stone [20] and White [21]. The main line of the proof is quite standard, as we study the asymptotic behavior of the flow in presence of a singularity, which (as it follows from the comparison principle) must happen in finite time. This kind of analysis is usually performed by means of a parabolic blow-up of the flow by a dilation factor (at every time) which diverges with the same order as the maximum of the curvature. This allows one to extract from the rescaled flow a sequence of curves with bounded curvature, and to get "easily" a subsequence converging to some limit curve containing the information on the asymptotic shape. Accordingly to the blow-up rate of the maximum of the curvature as $t$ approaches the singular time $T$, the possible singularities are then subdivided into in the so-called type $I$ and type II classes. Precisely, if there exists a constant $C$ such that

$$
\max _{\gamma_{t}} k^{2} \leq \frac{C}{2(T-t)} \quad \forall t \in[0, T),
$$

then the singularity is called of type I, if such constant does not exist, the singularity is called of type II.

Our analysis differs from the usual one insofar as we rescale the flow by the dilating factor $1 / 2(T-t)$ (which is the "natural" one for type I singularities), no matter what the rate of blow-up of the curvature is. Although this procedure gives no control on the maximum of the curvature along the blow-up, it allows to fully exploit Huisken's monotonicity formula [13] (Theorem 2.3) to get an $L_{\text {loc }}^{2}$-estimate on the curvature of a sequence of rescaled curves of the flow. This latter estimate implies the $C_{\mathrm{loc}}^{1}-$ convergence of a subsequence either to a straight line through the origin of $\mathbb{R}^{2}$ or to an embedded, closed, convex curve with positive curvature (actually to the unit circle in $\mathbb{R}^{2}$ ). We will prove that, either by a theorem of White [21] (Theorem 4.6) or as a consequence of an application of the interior estimates of Ecker and Huisken [6] (Theorem 2.4), the convergence to the straight line is not possible. On the other hand, if the sequence of dilated curves converges to the unit circle in $\mathbb{R}^{2}$, we will be able to exhibit a further sequence of curves in the rescaled flow which converges in the 
$C^{2}$-topology to an embedded, closed, convex curve with positive curvature, hence, such curves are definitely convex, thus proving Theorem 1.1.

We underline that the key fact, which allows us to pursue this "unitary" line of analysis without distinguishing the singularities into two types, is that in dimension one the uniform $L_{\text {loc }}^{2}$-control on the curvature implies the $C_{\text {loc }}^{1}$-subconvergence of the curves to some limit curve which, by a bootstrap argument starting from its $C^{1}$-regularity, can then be shown to be smooth.

The lack of such functional embedding (and thus the lack of the possibility to infer the smoothness of the limit along this line) is the main reason why our analysis is difficult to be pursued in higher dimensions, where the control of the mean curvature in $L_{\text {loc }}^{2}$ is not strong enough to give the $C_{\text {loc }}^{1}$-convergence of a subsequence of hypersurfaces to a limit. Nevertheless, very interesting results in this direction have been obtained by Ilmanen in dimension two [16] (which is, in some sense, the critical case). In particular, assuming the mean convexity of the surfaces, Ilmanen shows that it is possible to get a smooth limit for a suitable sequence of rescaled surfaces of the flow.

REMARK 1.3. Several techniques of this paper have been used by the authors, in collaboration with M. Novaga, also in the case of a network of three curves, concurrent at a triple point, moving by curvature to show that singularities cannot develop, see [18].

\section{Analytical and Geometrical Facts}

For any smooth, embedded, closed curve $\gamma: \mathbb{S}^{1} \rightarrow \mathbb{R}^{2}$ in the Euclidean plane, we will denote with $s$ its arclength parameter and with $v$ its inner unit normal vector field. The curvature of the curve will be $k=\left\langle\frac{d^{2} \gamma}{d s^{2}}, v\right\rangle$, where $\langle\cdot, \cdot\rangle$ is the Euclidean scalar product of $\mathbb{R}^{2}$.

Definition 2.1. Let $T>0$ and $\gamma: \mathbb{S}^{1} \times[0, T) \rightarrow \mathbb{R}^{2}$ be a smooth oneparameter family of closed and embedded curves. We say that $\gamma$ is a curvature flow of the initial curve $\gamma_{0}=\gamma(\cdot, 0)$ if for any time $t \in[0, T)$ and $\alpha \in \mathbb{S}^{1}$ there holds

$$
\partial_{t} \gamma(\alpha, t)=k(\alpha, t) v(\alpha, t)
$$

For every $t \in[0, T)$, we will use the notation $\gamma_{t}: \mathbb{S}^{1} \rightarrow \mathbb{R}^{2}$ for the curve given by $\gamma_{t}(x)=\gamma(x, t)$, that is, the evolving curve at time $t \in[0, T)$. 
In this section we collect some well known results about the curvature flow $\gamma$ of an initial closed and embedded curve $\gamma_{0}$ in $\mathbb{R}^{2}$ (see [19], for instance, for more details and proofs).

Proposition 2.2. If the initial curve $\gamma_{0}$ is smooth, the curvature flow problem has a unique smooth solution defined on a maximal time interval $[0, T)$ with $T<+\infty$ and, at every time $t \in[0, T)$, the curve $\gamma_{t}$ is a smooth embedding of $\mathbb{S}^{1}$ in $\mathbb{R}^{2}$.

The following evolution equations hold

$$
\begin{aligned}
& \partial_{t} d s=-k^{2} d s, \\
& \partial_{t} k=k_{s s}+k^{3}
\end{aligned}
$$

and

$$
\partial_{t} k_{s}=k_{s s s}+4 k^{2} k_{s}
$$

Moreover, there holds

$$
\max _{\gamma_{t}} k^{2} \geq \frac{1}{2(T-t)} \quad \forall t \in[0, T) .
$$

Huisken's monotonicity formula [13] holds.

TheOREM 2.3. For every $x_{0} \in \mathbb{R}^{2}$ we have

$$
\frac{d}{d t} \int_{\gamma_{t}} \frac{e^{-\frac{\left|x-x_{0}\right|^{2}}{4(T-t)}}}{\sqrt{4 \pi(T-t)}} d s=-\int_{\gamma_{t}} \frac{e^{-\frac{\left|x-x_{0}\right|^{2}}{4(T-t)}}}{\sqrt{4 \pi(T-t)}}\left|k+\frac{\left\langle x-x_{0} \mid v\right\rangle}{2(T-t)}\right|^{2} d s
$$

for any $t \in[0, T)$.

The following interior estimates were proven by Ecker and Huisken in [6], see also [14].

THEOREM 2.4. Let $e_{1}$ and $e_{2}$ be an orthonormal basis of $\mathrm{R}^{2}$ with respect to the standard Euclidean scalar product, let $\left\langle e_{1}\right\rangle=\operatorname{span}\left\{e_{1}\right\}$ and $\left\langle e_{2}\right\rangle=\operatorname{span}\left\{e_{2}\right\}$. For $x_{0} \in \mathbb{R}^{2}$ and $R>0$, suppose that in a ball $B_{2 R}\left(x_{0}\right)$ the curve $\gamma_{t}$, for $t \in[0, \tau)$, is a graph of a function over $\left\langle e_{1}\right\rangle$ and let $v=\left\langle v \mid e_{2}\right\rangle^{-1}>0$ at time $t=0$.

- Setting $\varphi(x, t)=R^{2}-\left|x-x_{0}\right|^{2}-2 t$, if $\varphi_{+}$denotes the positive part of $\varphi$, we have

$$
v(x, t) \varphi_{+}(x, t) \leq \sup _{x \in \gamma_{0}} v(x, 0) \varphi_{+}(x, 0)
$$


for every $t \in[0, \tau)$ and $x \in \gamma_{t}$, as long as $v(x, t)$ is defined everywhere on the support of $\varphi_{+}$.

- For arbitrary $\theta \in[0,1)$ we have the estimate

$$
\sup _{\gamma_{t} \cap B_{\theta R}\left(x_{0}\right)} k^{2} \leq C(1-\theta)^{-2}\left(\frac{1}{R^{2}}+\frac{1}{t}\right) \sup _{B_{R}\left(x_{0}\right) \times[0, \tau)} v^{4}
$$

for all $t \in[0, \tau)$, where the constant $C$ is independent of $t$ and $\gamma_{t}$.

Finally, we state a geometric result.

THEOREM 2.5 (Huisken's embeddedness measure [15]). For every $t \in[0, T)$, let $L_{t}$ be the length of the curve $\gamma_{t}$. We consider the function $\Phi_{t}: \gamma_{t} \times \gamma_{t} \rightarrow \mathbb{R}$ given by

$$
\Phi_{t}(x, y)= \begin{cases}\frac{\pi|x-y|}{L_{t}} / \sin \frac{\pi d_{t}(x, y)}{L_{t}} & \text { if } x \neq y \\ 1 & \text { if } x=y\end{cases}
$$

where $d_{t}(x, y)$ is the geodesic distance between $x$ and $y$ along $\gamma_{t}$.

Then, we define the following infimum, which, in the case of closed curves, is actually a minimum:

$$
E(t)=\inf _{x, y \in \gamma_{t}} \Phi_{t}(x, y)
$$

If the initial closed curve $\gamma_{0}$ is embedded, the function $E(t)$ is uniformly bounded below by a positive constant depending only on $\gamma_{0}$, for every $t \in[0, T)$.

Since the function $E(t)$ is positive if and only if $\gamma_{t}$ is embedded, an initial embedded and closed curve remains embedded during all the flow.

\section{The Density Function}

As in Stone [20], we consider the following Gaussian density function. For every $x_{0} \in \mathbb{R}^{2}$, we define

$$
\theta\left(x_{0}, t\right)=\int_{\gamma_{t}} \frac{e^{-\frac{\left|x-x_{0}\right|^{2}}{4(T-t)}}}{\sqrt{4 \pi(T-t)}} d s .
$$


By Huisken's monotonicity formula (2.2), we have

$$
\frac{\partial}{\partial t} \theta\left(x_{0}, t\right)=-\int_{\gamma_{t}} \frac{e^{-\frac{\left|x-x_{0}\right|^{2}}{4(T-t)}}}{\sqrt{4 \pi(T-t)}}\left|k+\frac{\left\langle x-x_{0} \mid v\right\rangle}{2(T-t)}\right|^{2} d s \leq 0
$$

for every $t \in[0, T)$. Hence, the limit $\Theta\left(x_{0}\right)=\lim _{t \rightarrow T} \theta\left(x_{0}, t\right)$ exists for every $x_{0} \in \mathbb{R}^{2}$ and, integrating, we get

$$
\theta\left(x_{0}, 0\right)-\Theta\left(x_{0}\right)=\int_{0}^{T} \int_{\gamma_{t}} \frac{e^{-\frac{\left|x-x_{0}\right|^{2}}{4(T-t)}}}{\sqrt{4 \pi(T-t)}}\left|k+\frac{\left\langle x-x_{0} \mid v\right\rangle}{2(T-t)}\right|^{2} d s d t .
$$

Letting $\mu(t)=\sup _{x_{0} \in \mathbb{R}^{2}} \theta\left(x_{0}, t\right)$, we can see that, as $\gamma_{t}$ is compact, this supremum is actually a maximum for any $t \in[0, T)$. Moreover, being a maximum of a family of positive and monotone nonincreasing functions, $\mu(t)$ is also monotone nonincreasing and positive. By looking at the right side of the monotonicity formula (2.2), we can finally notice that the functions $\theta\left(x_{0}, \cdot\right)$ are uniformly locally Lipschitz in time and we can conclude that the function $\mu$ is locally Lipschitz as well, hence differentiable at almost every $t \in[0, T)$.

Since $\mu$ is monotone, we can define $\Sigma=\lim _{t \rightarrow T} \mu(t) \in \mathbb{R}^{+}$, which clearly satisfies $\Sigma \geq \Theta\left(x_{0}\right)$ for every $x_{0} \in \mathbb{R}^{2}$.

For every $t \in[0, T)$ let $x_{t} \in \mathbb{R}^{2}$ be a point at which

$$
\mu(t)=\int_{\gamma_{t}} \frac{e^{-\frac{\left|x-x_{t}\right|^{2}}{4(T-t)}}}{\sqrt{4 \pi(T-t)}} d s
$$

that is, $x_{t}$ is a maximum point for the function $\theta(\cdot, t): \mathbb{R}^{2} \rightarrow \mathbb{R}^{+}$. We now make use of the following lemma to compute the derivative of the function $\mu$.

LEMMA 3.1 (Hamilton's Trick [12]). Let $M$ a Riemannian manifold and $u: M \times(0, T) \rightarrow \mathbb{R}$ be a $C^{1}$ function such that for every time $t$, there exists a value $\delta>0$ and a compact subset $K \subset M \backslash \partial M$ such that at every time $t^{\prime} \in(t-\delta, t+\delta)$ the maximum $u_{\max }\left(t^{\prime}\right)=\max _{p \in M} u\left(p, t^{\prime}\right)$ is attained at least at one point of $K$.

Then, $u_{\max }$ is a locally Lipschitz function in $(0, T)$ and at every differentiability time $t \in(0, T)$ we have

$$
\frac{d u_{\max }(t)}{d t}=\frac{\partial u(p, t)}{\partial t}
$$

where $p \in M$ is any inner point such that $u(\cdot, t)$ gets its maximum at $p$. 
Proposition 3.2. For every $t \in[0, T)$, let $x_{t} \in \mathbb{R}^{2}$ be as above, then

$$
\mu^{\prime}(t)=-\int_{\gamma_{t}} \frac{e^{-\frac{\left|x-x_{t}\right|^{2}}{4(T-t)}}}{\sqrt{4 \pi(T-t)}}\left|k+\frac{\left\langle x-x_{t} \mid v\right\rangle}{2(T-t)}\right|^{2} d s
$$

at almost every $t \in[0, T)$. Moreover,

$$
\mu(0)-\Sigma=\int_{0}^{T} \int_{\gamma_{t}} \frac{e^{-\frac{\left|x-x_{t}\right|^{2}}{4(T-t)}}}{\sqrt{4 \pi(T-t)}}\left|k+\frac{\left\langle x-x_{t} \mid v\right\rangle}{2(T-t)}\right|^{2} d s d t .
$$

Proof. Being $\theta(x, t)$ a $C^{1}$ function, Hamilton's trick applies. Hence, at every differentiability time $t \in[0, T)$, by formula (2.2) we have

$$
\mu^{\prime}(t)=-\int_{\gamma_{t}} \frac{e^{-\frac{\left|x-x_{t}\right|^{2}}{4(T-t)}}}{\sqrt{4 \pi(T-t)}}\left|k+\frac{\left\langle x-x_{t} \mid v\right\rangle}{2(T-t)}\right|^{2} d s .
$$

As the function $\mu$ is locally Lipschitz, we can integrate its derivative (which exists at almost every time) on every closed interval $[0, T-\varepsilon]$ and sending $\varepsilon$ to zero we get the second equality.

\section{Blowing-Up at a Singularity - The Proof of Grayson's Theorem}

As remarked in the introduction, we now rescale the curvature flow $\gamma: \mathbb{S}^{1} \times[0, T) \rightarrow \mathbb{R}^{2}$ in its maximal time interval $[0, T)$ without distinguishing between different types of singularities.

DEFINITION 4.1. We define the rescaled flow as follows,

$$
\widetilde{\gamma}(\alpha, r)=\frac{\gamma(\alpha, t(r))-x_{t(r)}}{\sqrt{2(T-t(r))}} \quad r=r(t)=-\frac{1}{2} \log (T-t),
$$

obtaining a smooth flow of embedded, closed curves $\widetilde{\gamma}_{r}: \mathbb{S}^{1} \rightarrow \mathbb{R}^{2}$, defined for $r \in\left[-\frac{1}{2} \log T,+\infty\right)$.

We rescale also formula (3.2) in order to get information on these curves. At almost every $r \in\left[-\frac{1}{2} \log T,+\infty\right)$ it holds 


$$
\begin{aligned}
\frac{d}{d r} \mu(t(r)) & =\mu^{\prime}(t(r)) 2(T-t(r)) \\
& =-\int \frac{e^{-\frac{\left|x-x_{t(r)}\right|^{2}}{4(T-t(r))}}}{\sqrt{4 \pi(T-t(r))}}\left|k \sqrt{2(T-t(r))}+\frac{\left\langle x-x_{t(r)} \mid v\right\rangle}{\sqrt{2(T-t(r))}}\right|^{2} d s \\
& =-\frac{1}{\sqrt{2 \pi}} \int_{\tilde{\gamma}_{r}} e^{-\frac{|y|^{2}}{2}}|\widetilde{k}+\langle y \mid \widetilde{v}\rangle|^{2} d s \leq 0 .
\end{aligned}
$$

Hence, integrating as before on $\left[-\frac{1}{2} \log T,+\infty\right)$, we get

$$
\begin{aligned}
\mu(0)-\Sigma & =\frac{1}{\sqrt{2 \pi}} \int_{\tilde{\gamma}_{-\frac{1}{2} \log T}} e^{-\frac{|y|^{2}}{2}} d s-\lim _{r \rightarrow+\infty} \frac{1}{\sqrt{2 \pi}} \int_{\tilde{\gamma}_{r}} e^{-\frac{|y|^{2}}{2}} d s \\
& =\frac{1}{\sqrt{2 \pi}} \int_{-\frac{1}{2} \log T} \int_{\tilde{\gamma}_{r}} e^{-\frac{|y|^{2}}{2}}|\widetilde{k}+\langle y \mid \widetilde{v}\rangle|^{2} d s d r<+\infty
\end{aligned}
$$

REMARK 4.2. Thanks to inequality (4.1), for every family of disjoint intervals $\left(a_{i}, b_{i}\right) \subset\left[-\frac{1}{2} \log T,+\infty\right)$ such that $\sum_{i \in \mathbb{N}}\left(b_{i}-a_{i}\right)=+\infty$, we can find a sequence $r_{i} \in\left(a_{i}, b_{i}\right)$ such that

$$
\lim _{i \rightarrow \infty} \frac{1}{\sqrt{2 \pi}} \int_{\tilde{\gamma}_{r_{i}}} e^{-\frac{|y|^{2}}{2}}|\widetilde{k}+\langle y \mid \widetilde{v}\rangle|^{2} d s=0
$$

and

$$
\lim _{i \rightarrow \infty} \frac{1}{\sqrt{2 \pi}} \int_{\tilde{\gamma}_{r_{i}}} e^{-\frac{|y|^{2}}{2}} d s=\lim _{i \rightarrow \infty} \mu\left(t\left(r_{i}\right)\right)=\Sigma .
$$

Clearly, the sequence $r_{i}$ is monotone increasing and diverges to $+\infty$.

We now state a technical lemma due to Stone which is crucial in order to commute limits and integrals.

LEMMA 4.3 (Stone [20]). Let $B_{R}$ a ball of radius $R>0$ in $\mathbb{R}^{2}$, then the following estimates hold uniformly in $r$ for the family of curves $\left\{\widetilde{\gamma}_{r}\right\}_{r \in\left[-\frac{1}{2} \log T,+\infty\right)}$.

1) There exist a constant $C$ independent of $B_{R}$ such that $\mathcal{H}^{1}\left(\widetilde{\gamma}_{r} \cap B_{R}\right) \leq$ $C e^{R^{2} / 2}$ where $\mathcal{H}^{1}$ is the one-dimensional Hausdorff measure in $\mathbb{R}^{2}$. 
2) For any $\varepsilon>0$ there exists a radius $R=R\left(\varepsilon\right.$, Length $\left.\left(\gamma_{0}\right), T\right)$ such that

$$
\int_{\tilde{\gamma}_{r} \backslash B_{R}(0)} e^{-\frac{|y|^{2}}{2}} d s \leq \varepsilon .
$$

Proposition 4.4. Let $r_{i}$ be a sequence as in Remark 4.2. Then, either for every $R>0$ the curves $\gamma_{r_{i}}$ definitely stay outside of the ball $B_{R}$, or there exists a smooth, complete, embedded curve $\widetilde{\gamma}_{\infty}$ (either closed or unbounded) satisfying $\widetilde{k}+\left\langle\widetilde{\gamma}_{\infty} \mid \widetilde{v}\right\rangle=0$, such that for a subsequence of $r_{i}$ (which we do not relabel) there holds $\gamma_{r_{i}} \rightarrow \widetilde{\gamma}_{\infty}$ in the $C_{\mathrm{loc}}^{1}$-topology.

Proof. Assume that we are in the second case. From the limit (4.2) and the estimate on the local length of the previous lemma, it follows that the sequence of curves $\widetilde{\gamma}_{r_{i}}$ has curvatures locally equibounded in $L^{2}$, as the term $|\langle y \mid \widetilde{v}\rangle|^{2}$ is clearly bounded in every ball of $\mathbb{R}^{2}$. Hence, we can extract a subsequence (not relabelled) that, after a possible reparametrization (in a constant multiple of the arclength of every curve), converges in $C_{\mathrm{loc}}^{1}$ to a complete limit curve $\widetilde{\gamma}_{\infty}$. Such curve satisfies $\widetilde{k}+\left\langle\widetilde{\gamma}_{\infty} \mid \widetilde{v}\right\rangle=0$, as the integral $\int_{\widetilde{\gamma}} e^{-\frac{\mid y^{2}}{2}}|\widetilde{k}+\langle y \mid \widetilde{v}\rangle|^{2} d s$ is lower semicontinuous under the $C_{\text {loc }}^{1}-$ convergence. The smoothness on the curve $\widetilde{\gamma}_{\infty}$ follows by a bootstrap argument applied to such relation.

Finally, the curve $\widetilde{\gamma}_{\infty}$ is embedded as the Huisken's embeddedness measure $E$ in Theorem 2.5 is scaling invariant and upper semicontinuous under the $C_{\text {loc }}^{1}$-convergence of curves, hence, such quantity is bounded below by a positive constant also for the limit curve $\widetilde{\gamma}_{\infty}$, which then must be embedded.

By the classification result of Abresch-Langer and Epstein-Weinstein (see [1] and [7] or [17, 19]), the limit curve $\widetilde{\gamma}_{\infty}$ can be either a line through the origin or the unit circle centered in the origin of $\mathbb{R}^{2}$. Actually, for our purposes, we only need the weaker conclusion that the limit curve is either a line through the origin or a closed, convex curve with positive curvature.

Lemma 4.5. Any smooth, complete, embedded curve $\sigma$ in $\mathbb{R}^{2}$ satisfying $k+\langle\sigma \mid v\rangle=0$ is either a line through the origin of $\mathbb{R}^{2}$ or a closed, convex curve with positive curvature.

Proof. The relation $k=-\langle\sigma \mid v\rangle$ implies that the curvature satisfies the ODE $k_{s}=k\langle\sigma \mid \tau\rangle$, where $\tau=\partial_{s} \sigma$ is a unit tangent vector. Suppose that 
at some point it holds $k=0$. By the ODE, at the same point holds also $k_{s}=0$ and hence, by the uniqueness theorem for ODE's, we conclude that $k$ is identically zero and we are dealing with a line $L$ which, as $\langle x \mid v\rangle=0$ for every $x \in L$, must contain the origin of $\mathbb{R}^{2}$.

We can now suppose that $k$ is always different from zero and, possibly after a reversion of the orientation of the curve, we can assume that $k>0$ at every point and thus that the curve is strictly convex.

The derivative of $|\sigma|^{2}$ with respect to the arclength parameter it is easily computed to be

$$
\partial_{s}|\sigma|^{2}=2\langle\sigma \mid \tau\rangle=2 k_{s} / k=2 \partial_{s} \log k .
$$

Thus we get $k=C e^{|\sigma|^{2} / 2}$, for some constant $C>0$ and it follows that $k$ is bounded from below by $C>0$.

We now consider a new coordinate $\alpha=\arccos \left\langle e_{1} \mid v\right\rangle$ which, by the convexity of the curve, is a good global parametrization (obviously, as for the arclength parameter $s$, the function $\alpha$ is only locally continuous since it "jumps" after a complete round).

Differentiating with respect to the arclength parameter we have $\partial_{s} \alpha=k$ and

$$
k_{\alpha}=k_{s} / k=\langle\sigma \mid \tau\rangle \quad k_{\alpha \alpha}=\frac{\partial_{s} k_{\alpha}}{k}=\frac{1+k\langle\sigma \mid v\rangle}{k}=\frac{1}{k}-k .
$$

Multiplying both sides of the last equation by $2 k_{\alpha}$ we get $\partial_{\alpha}\left[k_{\alpha}^{2}+k^{2}-\log k^{2}\right]=0$, which means that the quantity $k_{\alpha}^{2}+k^{2}-\log k^{2}$ is equal to some constant $Z$ along all the curve. Notice that such quantity $Z$ cannot be less than 1 and that, if $Z=1$, then $k$ must be constant and equal to one along the curve, which consequently must be the unit circle centered at the origin of $\mathbb{R}^{2}$.

If $Z>1$, it follows that $k$ is uniformly bounded from above. Hence, recalling that $k=C e^{|\sigma|^{2} / 2}$, the image of the curve is contained in a ball of $\mathbb{R}^{2}$ and, by the embeddedness and completeness hypotheses, the curve must be closed, simple and strictly convex, as $k>0$ at every point.

Since the second point of Lemma 4.3 implies that

$$
\lim _{i \rightarrow \infty} \frac{1}{\sqrt{2 \pi}} \int_{\tilde{\gamma}_{r_{i}}} e^{-\frac{|y|^{2}}{2}} d s=\frac{1}{\sqrt{2 \pi}} \int_{\tilde{\gamma}_{\infty}} e^{-\frac{|y|^{2}}{2}} d s,
$$

and, by equation (4.3), the left hand side is equal to $\Sigma$, we deduce that if we have a non empty limit curve then $\Sigma \geq 1$ and, by direct computation, we 
conclude that if $\Sigma>1$ then $\widetilde{\gamma}_{\infty}$ cannot be a line through the origin and hence it must be a closed, simple curve with positive curvature.

We now see that $\Sigma$ must be larger than one. To this aim we start with defining the set of reachable points of the curvature flow $\gamma$ by

$$
\mathcal{S}=\left\{x \in \mathbb{R}^{2} \mid \exists t_{i} \nearrow T \text { and } \alpha_{i} \in \mathbb{S}^{1} \text { such that } \gamma_{t_{i}}\left(\alpha_{i}\right) \rightarrow x\right\} .
$$

It is easy to see that $\mathcal{S}$ is non empty and compact. Moreover, if $x_{0} \notin \mathcal{S}$, the flow is clearly definitely far from $x_{0}$. If instead $x_{0} \in \mathcal{S}$, for every $t \in[0, T)$ the closed ball of radius $\sqrt{2(T-t)}$ and center $x_{0}$ intersects $\gamma_{t}$. Indeed, let $d_{t}=\min _{\alpha \in \mathbb{S}^{1}}\left|\varphi(\alpha, t)-x_{0}\right|$, that is, the Euclidean distance from $x_{0}$ to the curve $\gamma_{t}$.

The function $d_{t}:[0, T) \rightarrow \mathbb{R}$ is obviously locally Lipschitz and at a differentiability time with $d_{t}>0$, by Hamilton's trick, we have

$$
d_{t}^{\prime}(x)=\frac{\partial}{\partial t}\left|\gamma_{t}(\beta)-x_{0}\right| \geq \frac{k(\beta, t)\left\langle v(\beta, t) \mid \gamma_{t}(\beta)-x_{0}\right\rangle}{\left|\gamma_{t}(\beta)-x_{0}\right|}
$$

for any $\beta \in \mathbb{S}^{1}$ such that $d_{t}=\left|\gamma_{t}(\beta)-x_{0}\right|$.

By minimality, the closed ball $\bar{B}_{d_{t}}\left(x_{0}\right)$ intersects the curve $\gamma_{t}$ only on its boundary and the vector $\frac{\gamma_{t}(\beta)-x_{0}}{\left|\gamma_{t}(\beta, t)-x_{0}\right|}$ is parallel to the normal $v(\beta, t)$. An easy geometric argument shows then that

$$
\frac{k(q, t)\left\langle v(q, t) \mid \gamma_{t}(\beta)-x_{0}\right\rangle}{\left|\gamma_{t}(\beta)-x_{0}\right|} \geq-1 / d_{t} .
$$

Thus we conclude that, for almost every time $t \in[0, T)$ and if $d_{t} \neq 0$, we have

$$
d_{t}^{\prime}(x) \geq-1 / d_{t} .
$$

Integrating this differential inequality on the time interval $[t, \tau]$ we get $d_{t}^{2}-d_{\tau}^{2} \leq 2(\tau-t)$ and, by the hypothesis on $x_{0}$, we have $d_{t_{i}}^{2} \rightarrow 0$, hence

$$
d_{t}^{2}=\lim _{i \rightarrow \infty} d_{t}^{2}-d_{t_{i}}^{2} \leq \lim _{i \rightarrow \infty} 2\left(t_{i}-t\right)=2(T-t),
$$

which proves our claim.

Chosen $x_{0} \in \mathcal{S}$, we rescale the flow as follows, this time around the fixed point $x_{0}$,

$$
\bar{\gamma}(\alpha, r)=\frac{\gamma(\alpha, t(z))-x_{0}}{\sqrt{2(T-t(z))}} \quad z=z(t)=-\frac{1}{2} \log (T-t),
$$


obtaining a smooth flow of embedded, closed curves $\bar{\gamma}_{z}: \mathbb{S}^{1} \rightarrow \mathbb{R}^{2}$, defined for $z \in\left[-\frac{1}{2} \log T,+\infty\right)$. Notice that, by the above discussion, all these curves intersect the unit closed ball of $\mathbb{R}^{2}$.

Rescaling also formula (3.1), we have

$$
\theta\left(x_{0}, 0\right)-\Theta\left(x_{0}\right)=\frac{1}{\sqrt{2 \pi}} \int_{-\frac{1}{2} \log T}^{+\infty} \int_{\bar{\gamma}_{z}} e^{-\frac{|y|^{2}}{2}}|\bar{k}+\langle y \mid \bar{v}\rangle|^{2} d s d z<+\infty .
$$

Then, repeating the arguments in Remark 4.2 and Proposition 4.4, there exists a subsequence of $z_{i}$ (which we do not relabel) such that the curves $\gamma_{z_{i}}$ converge in the $C_{\text {loc }}^{1}$-topology to a smooth, complete, embedded curve $\bar{\gamma}_{\infty}$, which is not empty (the limit curve also must intersect the unit closed ball of $\mathbb{R}^{2}$ ), satisfies $\bar{k}+\left\langle\bar{\gamma}_{\infty} \mid \bar{v}\right\rangle=0$ and

$$
\frac{1}{\sqrt{2 \pi}} \int_{\bar{\gamma}_{\infty}} e^{-\frac{|y|^{2}}{2}} d s=\lim _{i \rightarrow \infty} \frac{1}{\sqrt{2 \pi}} \int_{\bar{\gamma}_{z_{i}}} e^{-\frac{|y|^{2}}{2}} d s=\Theta\left(x_{0}\right) .
$$

Hence, by Lemma 4.5 and a direct computation, it follows that $\Theta\left(x_{0}\right) \geq 1$, hence $\Sigma \geq 1$. Moreover, if we assume that $\Sigma=1$ we conclude that $\Theta\left(x_{0}\right)=1$ for every $x_{0} \in \mathcal{S}$ and the subsequence of rescaled curves $\gamma_{z_{i}}$ converges in the $C_{\text {loc }}^{1}$-topology to a line through the origin of $\mathbb{R}^{2}$.

The following general local regularity theorem of White [21] (holding for the mean curvature flow in any dimension) can now be applied.

THEOREM 4.6. There exists a constant $\varepsilon>0$ such that if a point $x_{0} \in \mathcal{S}$ satisfies $\Theta\left(x_{0}\right)<1+\varepsilon$, then there exists a radius $R>0$ such that in $B_{R}\left(x_{0}\right) \times[0, T) \subset \mathbb{R}^{2} \times \mathbb{R}$ the curvature is uniformly bounded.

Clearly, this theorem contradicts the assumption $\Sigma=1$, as (by a compactness argument on the set $\mathcal{S}$ ) it implies that the curvature is uniformly bounded when $t \rightarrow T$ and this is impossible since estimate (2.1) holds.

In the special case of simple curves, the exclusion of the case $\Sigma=1$ can be actually deduced as follows also by means of the interior estimates of Ecker and Huisken (Theorem 2.4).

Chosen any $x_{0} \in \mathcal{S}$, by the $C_{\text {loc }}^{1}$-convergence of the rescaled curves $\widetilde{\gamma}_{z_{i}}$ to a line through the origin of $\mathbb{R}^{2}$, for every $R>2$ there is a sequence of times $t_{i} \nearrow T$ and a line $\mathrm{L}$ passing for $x_{0}$ such that every curve $\gamma_{t_{i}}$ is a graph over $\mathrm{L}$ in the ball $B_{R \sqrt{2\left(T-t_{i}\right)}}\left(x_{0}\right)$. 
Supposing that $x_{0}=0$ and that $\mathrm{L}$ is $\left\langle e_{1}\right\rangle$ in $\mathbb{R}^{2}$, the pieces of curves $\gamma_{t} \cap B_{R \sqrt{2\left(T-t_{i}\right)}}$ can be represented, at least for small times after $t_{i}$, as a graphs of a time dependent function. Moreover, choosing $i$ large enough, for every $x \in \gamma_{t_{i}} \cap B_{R \sqrt{2\left(T-t_{i}\right)}}$, the quantity $v(x, t)=\left\langle v(x, t) \mid e_{2}\right\rangle^{-1}$ is arbitrarily close to one at time $t=t_{i}$.

As the circle $\partial B_{\sqrt{2(T+\varepsilon-t)}}$ is moving by curvature and, choosing $\varepsilon>0$ small enough, at time $t=t_{i}$ it is contained in the ball $B_{R \sqrt{2\left(T-t_{i}\right)}}$, by the Sturmian theorem of Angenent in [4, Proposition 1.2] and [3, Section 2] (see [2] for the proof), we have that the number of intersections (which is two at time $t_{i}$ ) between the curve $\gamma_{t}$ and the circle $\partial B_{\sqrt{2(T+\varepsilon-t)}}$ cannot increase in time. On the other hand, the number of intersections can also not decrease, otherwise the whole curve $\gamma_{t}$ would be definitely contained inside $B \sqrt{2(T+\varepsilon-t)}$, in contradiction with the fact that the limit of the rescaled curves $\widetilde{\gamma}_{z_{i}}$ is the unbounded line $L$. This argument shows that it is not possible that other parts of the moving curve $\gamma_{t}$ "get into" the ball $B \sqrt{2(T+\varepsilon-t)}$ at some time $t>t_{i}$. Consequently, the only reason for which $\gamma_{t} \cap B \sqrt{2(T+\varepsilon-t)}$ can possibly stop being a graph is that the tangent vector to such graph becomes vertical at some time, or, equivalently, that the function $v$ is not bounded.

Inequality (2.3) excludes this fact if the quantity $v$ at time $t_{i}$ is small enough. Hence, with a suitable choice of one of the times $t_{i}$, by estimate (2.4), the curvature of $\gamma_{t}$ for $t \in\left[t_{i}, T\right)$ is bounded in the ball $B_{\sqrt{2(T+\varepsilon-t)}}$, in particular it is bounded in $B_{\sqrt{2 \varepsilon}} \subset B_{\sqrt{2(T+\varepsilon-t)}}$ for every $t \in\left[t_{i}, T\right)$.

By the same argument above, this implies that the curvature is uniformly bounded as $t \rightarrow T$, contradicting inequality (2.1).

REMARK 4.7. We underline that the key point in getting a bound on the curvature by means of this argument is the $C_{\mathrm{loc}}^{1}$-convergence of the rescaled curves to a line (by the $L^{2}$ bound on the curvature), which does not follow in higher dimensions.

Once established that $\Sigma>1$, getting back to the original rescaling as in Proposition 4.4, we can assume that the sequence of rescaled curves $\widetilde{\gamma}_{r_{i}}$ converges in $C_{\text {loc }}^{1}$ to a closed, convex curve $\widetilde{\gamma}_{\infty}$ in $\mathbb{R}^{2}$ with positive curvature. Hence, as the curve $\widetilde{\gamma}_{\infty}$ is compact, the convergence is actually in $C^{1}$ with equibounded curvatures in $L^{2}$. 
Grayson's Theorem is then a consequence of the following proposition, which clearly implies that the curves $\gamma_{t\left(q_{i}\right)}$ definitively have positive curvature and hence are convex.

Proposition 4.8. There exists a sequence $q_{i} \nearrow+\infty$ such that the rescaled curves $\widetilde{\gamma}_{q_{i}}$ converge in the $C^{2}$-topology to a closed, convex curve with positive curvature.

Proof. Fixing $i \in \mathbb{N}$ and letting $\rho=r-r_{i}<1$, as $r=-\frac{1}{2} \log (T-t)$, we compute

$$
\begin{aligned}
\frac{d}{d r} \int_{\widetilde{\gamma}_{r}}\left(\widetilde{k}^{2}+\rho \widetilde{k}_{s}^{2}\right) d s= & 2(T-t) \frac{d}{d t} \int_{\gamma_{t}} \sqrt{2(T-t)} k^{2} d s+\int_{\widetilde{\gamma}_{r}} \widetilde{k}_{s}^{2} d s \\
& +2(T-t) \rho \frac{d}{d t} \int_{\gamma_{t}}(\sqrt{2(T-t)})^{3} k_{s}^{2} d s \\
= & -\sqrt{2(T-t)} \int_{\gamma_{t}} k^{2} d s+(\sqrt{2(T-t)})^{3} \int_{\gamma_{t}}\left(2 k k_{s s}+k^{4}\right) d s \\
& +\int_{\widetilde{\gamma}_{r}} \widetilde{k}_{s}^{2} d s-3(\sqrt{2(T-t)})^{3} \rho \int_{\gamma_{t}} k_{s}^{2} d s \\
& +(\sqrt{2(T-t)})^{5} \rho \int_{\gamma_{t}}\left(2 k_{s} k_{s s s}+7 k^{2} k_{s}^{2}\right) d s \\
= & \int_{\widetilde{\gamma}_{r}}\left[-\widetilde{k}^{2}+2 \widetilde{k} \widetilde{k}_{s s}+\widetilde{k}^{4}+\widetilde{k}_{s}^{2}-3 \rho \widetilde{k}_{s}^{2}+2 \rho \widetilde{k}_{s} \widetilde{k}_{s s s}+7 \rho \widetilde{k}^{2} \widetilde{k}_{s}^{2}\right] d s,
\end{aligned}
$$

where we used the formulas in Proposition 2.2.

Integrating by parts and by Peter-Paul inequality, we have

$$
\int_{\tilde{\gamma}_{r}} \widetilde{k}^{2} \widetilde{k}_{s}^{2} d s=\frac{1}{3} \int_{\widetilde{\gamma}_{r}} \partial_{s}\left(\widetilde{k}^{3}\right) \widetilde{k}_{s} d s=-\frac{1}{3} \int_{\widetilde{\gamma}_{r}} \widetilde{k}^{3} \widetilde{k}_{s s} d s \leq \frac{1}{6} \int_{\widetilde{\gamma}_{r}} \widetilde{k}^{6}+\widetilde{k}_{s s}^{2} d s
$$

and

$$
\begin{aligned}
\frac{d}{d r} \int_{\widetilde{\gamma}_{r}}\left(\widetilde{k}^{2}+\rho \widetilde{k}_{s}^{2}\right) d s & \leq \int_{\widetilde{\gamma}_{r}}\left[-\widetilde{k}_{s}^{2}+\widetilde{k}^{4}-\widetilde{k}^{2}-3 \rho \widetilde{k}_{s}^{2}-2 \rho \widetilde{k}_{s s}^{2}+7 \rho\left(\widetilde{k}^{6}+\widetilde{k}_{s s}^{2}\right) / 6\right] d s \\
& \leq \int_{\tilde{\gamma}_{r}}\left(-\widetilde{k}_{s}^{2}+\widetilde{k}^{4}+3 \rho \widetilde{k}^{6}\right) d s .
\end{aligned}
$$


Thus, the following interpolation inequalities, holding for any closed curve of length $L$ in the plane (see Aubin [5, page 93]), $\|\widetilde{k}\|_{L^{4}}^{4} \leq C\left\|\widetilde{k}_{s}\right\|_{L^{2}}\|\widetilde{k}\|_{L^{2}}^{3}+\frac{C}{L}\|\widetilde{k}\|_{L^{2}}^{4} \quad$ and $\quad\|\widetilde{k}\|_{L^{6}}^{6} \leq C\left\|\widetilde{k}_{s}\right\|_{L^{2}}^{2}\|\widetilde{k}\|_{L^{2}}^{4}+\frac{C}{L^{2}}\|\widetilde{k}\|_{L^{2}}^{6}$, imply (also by means of the Young inequality)

$$
\int_{\tilde{\gamma}_{r}} \widetilde{k}^{4} d s \leq \frac{1}{2} \int_{\tilde{\gamma}_{r}} \widetilde{k}_{s}^{2} d s+\frac{1}{2}\left(\int_{\tilde{\gamma}_{r}} \widetilde{k}^{2} d s\right)^{3}+\left(\int_{\tilde{\gamma}_{r}} \widetilde{k}^{2} d s\right)^{3}+\frac{C}{L^{3}\left(\widetilde{\gamma}_{r}\right)}
$$

and

$$
3 \rho \int_{\widetilde{\gamma}_{r}} \widetilde{k}^{6} d s \leq\left(\rho \int_{\widetilde{\gamma}_{r}} \widetilde{k}_{s}^{2} d s\right)^{3}+2\left(\int_{\widetilde{\gamma}_{r}} \widetilde{k}^{2} d s\right)^{3}+\frac{C}{L^{2}\left(\widetilde{\gamma}_{r}\right)}\left(\int_{\tilde{\gamma}_{r}} \widetilde{k}^{2} d s\right)^{3} .
$$

Hence, as we know that $L\left(\widetilde{\gamma}_{r}\right) \geq \int_{\tilde{\gamma}_{r}} e^{-\frac{|y|^{2}}{2}} d s \geq \sqrt{2 \pi}$ and $\rho<1$, we conclude

$$
\begin{aligned}
\frac{d}{d r} \int_{\tilde{\gamma}_{r}}\left(\widetilde{k}^{2}+\rho \widetilde{k}_{s}^{2}\right) d s \leq & \int_{\tilde{\gamma}_{r}}\left(-\widetilde{k}_{s}^{2}+\widetilde{k}_{s}^{2} / 2\right) d s+C\left(\int_{\tilde{\gamma}_{r}} \widetilde{k}^{2} d s\right)^{3}+C \\
& +\left(\rho \int_{\tilde{\gamma}_{r}} \widetilde{k}_{s}^{2} d s\right)^{3}+C\left(\int_{\tilde{\gamma}_{r}} \widetilde{k}^{2} d s\right)^{3} \\
\leq & C\left(\int_{\tilde{\gamma}_{r}} \widetilde{k}^{2} d s\right)^{3}+\left(\rho \int_{\tilde{\gamma}_{r}} \widetilde{k}_{s}^{2} d s\right)^{3}+C \\
\leq & C\left(\int_{\tilde{\gamma}_{r}}\left(\widetilde{k}^{2}+\rho \widetilde{k}_{s}^{2}\right) d s\right)^{3}+C
\end{aligned}
$$

for a constant $C$ independent of $r \geq r_{i}$ and $i \in \mathbb{N}$.

Integrating this differential inequality for the quantity $Q_{i}(r)=$ $\int_{\tilde{\gamma}_{r}}\left(\widetilde{k}^{2}+\left(r-r_{i}\right) \widetilde{k}_{s}^{2}\right) d s$ over the interval $\left[r_{i}, r_{i}+2 \delta\right]$, it is easy to see that if $\delta>0$ is small enough, we have

$$
Q_{i}(r) \leq C\left(\delta, Q_{i}\left(r_{i}\right)\right)=C\left(\delta, \int_{\tilde{\gamma}_{r_{i}}} \widetilde{k}^{2} d s\right)=C(\delta),
$$


for every $r \in\left[r_{i}, r_{i}+2 \delta\right]$, as the curves $\widetilde{\gamma}_{r_{i}}$ have uniformly bounded curvature in $L^{2}$. Hence, if $r \in\left[r_{i}+\delta, r_{i}+2 \delta\right]$ we have the estimate

$$
\int_{\tilde{\gamma}_{r}}\left(\widetilde{k}^{2}+\delta \widetilde{k}_{s}^{2}\right) d s \leq \int_{\tilde{\gamma}_{r}}\left(\widetilde{k}^{2}+\left(r-r_{i}\right) \widetilde{k}_{s}^{2}\right) d s \leq C(\delta)
$$

which implies

$$
\int_{\tilde{\gamma}_{r}}\left(\widetilde{k}^{2}+\widetilde{k}_{s}^{2}\right) d s \leq C(\delta)+\frac{C(\delta)}{\delta}
$$

for every $r \in\left[r_{i}+\delta, r_{i}+2 \delta\right]$ and a constant $C(\delta)$ independent of $i \in \mathbb{N}$.

We can now find as before, using again Remark 4.2, a sequence of values $q_{i} \in\left[r_{i}+\delta, r_{i}+2 \delta\right]$ such that

$$
\lim _{i \rightarrow \infty} \frac{1}{\sqrt{2 \pi}} \int_{\tilde{\gamma}_{q_{i}}} e^{-\frac{|y|^{2}}{2}}|\widetilde{k}+\langle y \mid \widetilde{v}\rangle|^{2} d s=0 .
$$

and

$$
\lim _{i \rightarrow \infty} \frac{1}{\sqrt{2 \pi}} \int_{\tilde{\gamma}_{q_{i}}} e^{-\frac{|y|^{2}}{2}} d s=\lim _{i \rightarrow \infty} \mu\left(t\left(q_{i}\right)\right)=\Sigma>1 .
$$

As this new sequence of rescaled curves $\widetilde{\gamma}_{q_{i}}$ satisfies the local length estimate of Lemma 4.3 and $\widetilde{k}$ and $\widetilde{k}_{s}$ are uniformly bounded in $L^{2}$, arguing as in Proposition 4.4 and in the subsequent discussion, we can extract a subsequence (not relabelled) that converges in $C^{2}$ to a limit curve which cannot be a line and hence, by Lemma 4.5, must be a closed curve with positive curvature.

Acknowledgments. Annibale Magni was partially supported by the DFG Collaborative Research Center SFB/Transregio 71. Carlo Mantegazza was partially supported by the Italian FIRB Ideas "Analysis and Beyond".

\section{REFERENCES}

[1] U. Abresch - J. LANGER, The normalized curve shortening flow and homothetic solutions, J. Diff. Geom. 23 (1986), no. 2, pp. 175-196.

[2] S. Angenent, The zero set of a solution of a parabolic equation, J. Reine Angew. Math. 390 (1988), pp. 79-96. 
[3] S. Angenent, On the formation of singularities in the curve shortening flow, J. Diff. Geom. 33 (1991), pp. 601-633.

[4] S. Angenent, Parabolic equations for curves on surfaces. II. Intersections, blow-up and generalized solutions, Ann. of Math. (2) 133 (1991), no. 1, pp. 171-215.

[5] T. Aubin, Some nonlinear problems in Riemannian geometry, SpringerVerlag, 1998.

[6] K. EcKer - G. Huisken, Interior estimates for hypersurfaces moving by mean curvature, Invent. Math. 105 (1991), no. 3, pp. 547-569.

[7] C. L. Epstein - M. I. Weinstein, A stable manifold theorem for the curve shortening equation, Comm. Pure Appl. Math. 40 (1987), no. 1, pp. 119-139.

[8] M. GAGE, An isoperimetric inequality with applications to curve shortening, Duke Math. J. 50 (1983), no. 4, pp. 1225-1229.

[9] M. GaGE, Curve shortening makes convex curves circular, Invent. Math. 76 (1984), pp. 357-364.

[10] M. GaGE - R. S. Hamilton, The heat equation shrinking convex plane curves, J. Diff. Geom. 23 (1986), pp. 69-95.

[11] M. A. Grayson, The heat equation shrinks embedded plane curves to round points, J. Diff. Geom. 26 (1987), pp. 285-314.

[12] R. S. Hamilton, Four-manifolds with positive curvature operator, J. Diff. Geom. 24 (1986), no. 2, pp. 153-179.

[13] G. Huisken, Asymptotic behavior for singularities of the mean curvature flow, J. Diff. Geom. 31 (1990), pp. 285-299.

[14] G. Huisken, Local and global behaviour of hypersurfaces moving by mean curvature, Proc. Sympos. Pure Math 54 (1993), pp. 175-191.

[15] G. Huisken, A distance comparison principle for evolving curves, Asian J. Math. 2 (1998), pp. 127-133.

[16] T. Ilmanen, Singularities of mean curvatureflow of surfaces, http:// www.math.ethz.ch/ ilmanen/papers/sing.ps, 1995.

[17] A. Magni - C. Mantegazza, Curves homothetically shrinking by curvature, CvGmt Preprint Server - http://cvgmt.sns.it, 2009.

[18] A. Magni - C. Mantegazza - M. Novaga, Motion by curvature of planar networks II, ArXiv Preprint Server - http://arxiv.org, 2013.

[19] C. Mantegazza, Lecture notes on mean curvature flow, Progress in Mathematics, vol. 290, Birkhäuser/Springer Basel AG, Basel, 2011.

[20] A. Stone, A density function and the structure of singularities of the mean curvature flow, Calc. Var. Partial Differential Equations 2 (1994), 443-480.

[21] B. White, A local regularity theorem for mean curvature flow, Ann. of Math. (2) 161 (2005), no. 3, 1487-1519.

Manoscritto pervenuto in redazione il 9 Aprile 2013. 
